\title{
Temporal and Spatial Regulation of Targeting Aurora B to the Inner Centromere
}

\author{
Y. WATANABE \\ Laboratory of Chromosome Dynamics, Institute of Molecular and Cellular Biosciences, \\ University of Tokyo, Yayoi, Tokyo 113-0032, Japan \\ Correspondence: ywatanab@iam.u-tokyo.ac.jp
}

\begin{abstract}
Successful partitioning of chromosomes in mitosis relies on the bipolar attachment of sister chromatids at metaphase. For this biorientation, the chromosomal passenger complex (CPC), composed of catalytic kinase Aurora B and regulatory components (INCENP, Survivin, and Borealin), must be localized at the center of paired kinetochores, the site called the inner centromere. It is largely unknown what defines the inner centromere and how the CPC is targeted to this site. Recent studies point out that the shugoshin protein (SGO), originally identified as a cohesin protector, also acts as a conserved centromeric adapter of the CPC. Phosphorylation of the CPC by Cdk1 promotes direct binding with shugoshin, thus explaining how the $\mathrm{CPC}$ is targeted to the centromere in a timely manner at prometaphase during the cell cycle. Moreover, the phosphorylation of histone $\mathrm{H} 3$ threonine 3 (H3-pT3) mediated by Haspin cooperates with Bub1-mediated H2A-S121 phosphorylation in targeting the CPC to the inner centromere. H3-pT3 promotes nucleosome binding of Survivin, whereas H2A-pS121 facilitates the binding of shugoshin. Haspin colocalizes with cohesin by associating with Pds5, a cohesin-binding protein, and Bub1 localizes at kinetochores. Thus, the inner centromere is defined by the spatial intersection of two histone marks mediated by cohesin- and kinetochore-associated kinases.
\end{abstract}

For the proper partition of chromosomes to daughter cells, sister chromatids must be held together before mitotic chromosome segregation. This cohesion of sister chromatids is established during DNA replication dependent on the cohesin complex and is maintained until metaphase when sister kinetochores attach to microtubules (MTs) emanating from the opposite spindle poles (bipolar attachment) (Nasmyth et al. 2000). In animal mitotic cells, however, most cohesin dissociates from the chromosome arms during prophase (called "prophase pathway"), in a manner dependent on the phosphorylation of cohesin by mitotic kinases such as Polo-like kinase (Losada et al. 2002; Hauf et al. 2005). However, centromeric cohesin is retained until metaphase through the function of shugoshin (Sgo/MEI-S332) (Lee et al. 2005; Watanabe 2005). This shugoshin-dependent centromeric protection is essential for mitotic chromosome segregation in mammalian cells. In most organisms, shugoshin is required for meiotic chromosome segregation, where the stepwise dissociation of cohesin is more crucial (Miyazaki and Orr-Weaver 1994; Watanabe 2005). Shugoshin collaborates with protein phosphatase 2A (PP2A) to prevent phosphorylation of cohesin and, thereby, its dissociation (Kitajima et al. 2006; Riedel et al. 2006; Tang et al. 2006). Although shugoshin was first characterized as a protector of centromeric cohesin (Kitajima et al. 2004; Marston et al. 2004; Rabitsch et al. 2004), a study in budding yeast suggested that it was also involved in sensing the lack of tension on mitotic chromosomes (Indjeian et al. 2005).

Accurate segregation of chromosomes during cell division requires sister chromatids to be captured by MTs from the opposite spindle poles (bipolar attachment or biorientation). This is accomplished by a trial-and-error process of kinetochore-microtubule (KT-MT) attachment that is essential and relies largely on the $\mathrm{CPC}$, which includes catalytic kinase Aurora B and regulatory subunits Borealin, Survivin, and INCENP and locates at centromeres (Hauf et al. 2003; Cheeseman et al. 2006; DeLuca et al. 2006; Ciferri et al. 2008; Tanaka 2008). In the absence of tension across centromeres, Aurora B phosphorylates the MT-anchoring kinetochore proteins, thereby destabilizing the attachment, whereas the bipolar attachment of kinetochores produces a tension that brings about the spatial separation of kinetochores from Aurora B, thereby stabilizing the attachment (Fig. 1) (Pinsky and Biggins 2005; Tanaka 2008; Liu et al. 2009). Thus, Aurora B must be targeted and anchored precisely at the center of paired kinetochores, a site called the inner centromere (Ruchaud et al. 2007; Kelly and Funabiki 2009).

\section{SHUGOSHIN REQUIRED FOR CPC TARGETING TO CENTROMERES}

Fission yeast possess two shugoshin-like proteins, Sgo1 and Sgo2, both of which localize at the pericentromeric heterochromatin region, the site enriched with cohesin and crucial for centromeric cohesion. Sgol is meiosis I specific and has a crucial role in protecting centromeric cohesin during meiosis I, whereas Sgo2 is ubiquitously expressed and required for faithful chromosome segregation in mitosis and meiosis (Kitajima et al. 2004; Rabitsch et al. 2004). Sgo2 associates closely with Bir1/Survivin and is required for the full localization of the CPC at centromeres (Kawashima et al. 2007; Vanoosthuyse et al. 


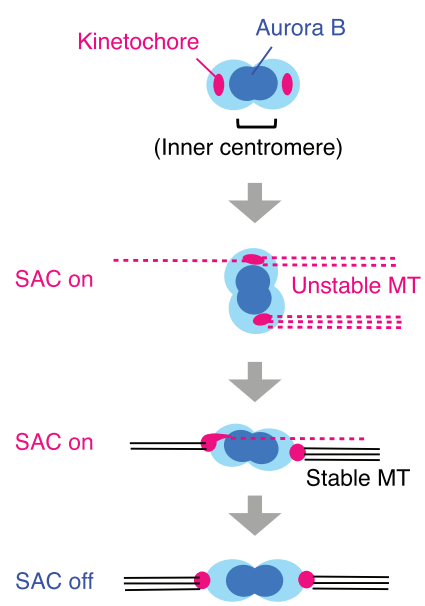

Figure 1. Establishment of bipolar attachment. Kinetochore-assembling core centromere (pink globule) protrudes from inner centromere, the site where Aurora B kinase accumulates and destabilizes kinetochore-microtubule attachment. Therefore, microtubule (MT) attachment to the kinetochore within the AuroraB-active region (dark blue and light blue) is unstable (dashed pink line), whereas MT attachment far from Aurora B activity is stable (black line). Spindle assembly checkpoint is activated when unstable attachment is disconnected but is inactivated when all attachment is bipolar and thus stabilized (bottom).

2007). The overall phenotypes of sgo $2 \Delta$, not only during mitosis but also during meiosis, are explainable by the reduced activity of Aurora B. These results imply that in addition to its protective function, shugoshin has a crucial role in promoting the centromeric localization of the CPC, thereby ensuring bipolar attachment of kinetochores.

The requirement of shugoshin for CPC targeting to centromeres has also been shown in Xenopus (Rivera and Losada 2009). Although depletion of hSgo1, a canonical shugoshin in human mitotic cells, causes little defect in CPC localization, depletion of both shugoshin-like proteins hSgo 1 and hSgo 2 abolishes CPC localization to centromeres, validating that the second role of shugoshin is also conserved in mammals (Tsukahara et al. 2010). Centromeric localization of shugoshin is also influenced by the $\mathrm{CPC}$, implying that centromere targeting of shugoshin and the CPC is interdependent (Resnick et al. 2006; Kawashima et al. 2007; Vanoosthuyse et al. 2007). Thus, whereas shugoshin was initially identified as a cohesin protector, the same or related protein acts as the centromeric CPC adapter in eukaryotes.

\section{CDK1-DEPENDENT CPC PHOSPHORYLATION REQUIRED FOR CENTROMERE TARGETING}

The cyclin-dependent kinase 1 (Cdk1)-cyclin B complex functions as a cell cycle engine to promote mitotic progression. The newly isolated fission yeast cyclin B mutant $c d c 13-M 7$ shows a marked defect in chromosome biorientation but not in mitotic entry, suggesting that the Cdk1-cyclin B complex specifically targets the regulation of chromosome biorientation in addition to cell cycle pro-

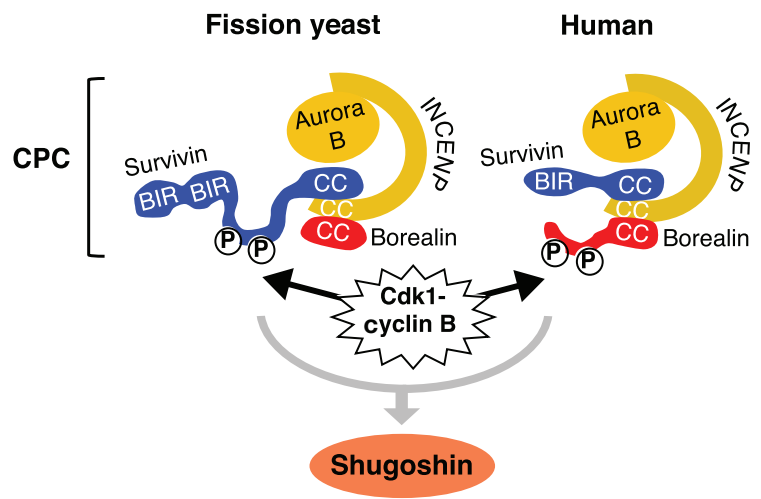

Figure 2. Interaction between the CPC and shugoshin. Phosphorylation of fission yeast Survivin or human Borealin by Cdk1 promotes CPC binding to coiled-coil region of shugoshin. Intertwined configuration of Survivin, Borealin, and INCENP is based on the crystallographic study of the complex (Jeyaprakash et al. 2007). (Modified, with permission, from Tsukahara et al. 2010 [Nature Publishing Group].)

gression (Tsukahara et al. 2010). Accordingly, fission yeast Survivin/Bir1 is phosphorylated by $\mathrm{Cdc} 2$ in mitosis, and the nonphosphorylatable bir 1-8A mutant impairs CPC localization and its function at centromeres. In contrast, the phosphomimetic bir 1-8D mutant shows intact CPC targeting to centromeres and, crucially, suppresses the biorientation defect of the $c d c 13-M 7$ mutant. Thus, phosphorylation of Bir1 is important for targeting the CPC to centromeres in prometaphase (Tsukahara et al. 2010).

The amino-terminal half of Bir1 (Bir1-N), which contains a CDK phosphorylation cluster, binds Sgo2, a centromeric CPC adapter, and phosphorylation of Bir1 is required for this interaction (Fig. 2). As in fission yeast, the inhibition of human cyclin B causes defects in chromosome biorientation (Chen et al. 2008). Consistently, the Cdk1-dependent phosphorylation of hBorealin (rather than Survivin in this case) promotes centromere targeting through association with both hSgo1 and hSgo2 (Tsukahara et al. 2010). Although the CPC subunit that interacts with shugoshin thus moves between yeast and metazoa, the overall regulation of $\mathrm{CPC}$ targeting to the centromere by Cdk1 is conserved, explaining how the cell cycle is linked to CPC targeting to centromeres and chromosome biorientation in eukaryotes (Fig. 2).

\section{BUB1-DEPENDENT H2A PHOSPHORYLATION REQUIRED FOR SHUGOSHIN LOCALIZATION}

Shugoshin localization at centromeres depends on the conserved SAC protein kinase Bub1, which is also required for proper chromosome segregation (Kitajima et al. 2004, 2005; Boyarchuk et al. 2007). Although several Bub1 substrate candidates have been suggested, the canonical substrate remained unknown more than 15 years after its discovery, even in genetically tractable organisms such as yeast. Recently, a biochemical approach in fission yeast identified serine 121 of histone $2 \mathrm{~A}(\mathrm{H} 2 \mathrm{~A})$ as a Bub1 substrate (Kawashima et al. 2010). Crucially, the $h 2 a-S 121 A$ 


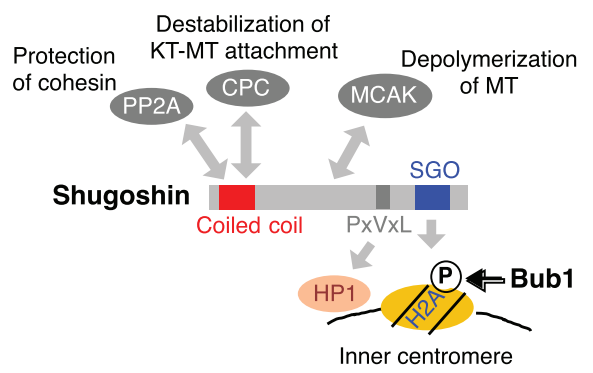

Figure 3. Shugoshin action. Shugoshin, which can associate with PP2A, CPC, and MCAK, is targeted to the nucleosome at inner centromeres through the SGO motif, which binds the nucleosome only when $\mathrm{H} 2 \mathrm{~A}$ is phosphorylated by Bub1. Localization of fission yeast Sgol and human hSgol at inner centromere is also mediated by interaction with the heterochromatin protein Swi6/HP1 through the PxVxL motif (Yamagishi et al. 2008). (KT-MT) Kinetochore-microtubule.

mutant, in which all cellular H2A-S121 is replaced by alanine, phenocopies the bubl kinase-dead (bubl-KD) mutant in losing the centromeric localization of shugoshin proteins, indicating that $\mathrm{H} 2 \mathrm{~A}-\mathrm{S} 121$ is the canonical cellular substrate of Bub1. Artificial tethering of shugoshin to centromeres largely restores the $h 2 a-S 121 A-$ or bubl-KDrelated defects in chromosome segregation. The Bub1H2A-shugoshin pathway is also conserved in human cells (Kawashima et al. 2010).

Shugoshin family proteins, although conserved among all eukaryotes, share limited amino acid sequence similarities in the coiled-coil region near the amino terminus and the basic region near the carboxyl terminus (Fig. 3) (Kitajima et al. 2004). This basic sequence, denoted as the SGO (shugoshin) motif, mediates the association with nucleosomes containing H2A-pS121 (Fig. 3, also see Fig. 4A) (Kawashima et al. 2010). In contrast, the coiled-coil region is responsible for the interaction with PP2A and the CPC and thereby for their recruitment to centromeres (Kawashima et al. 2007; Tsukahara et al. 2010). Recent studies in human cells have revealed that the middle nonconserved region of $\mathrm{hSgo} 2$ but not hSgol additionally binds MCAK, an MT depolymerase that has a key role in correcting attachment, to recruit it to centromeres (Tanno et al. 2010). Thus, shugoshin acts as a molecular hub, linking several centromeric proteins to the nucleosome at the inner centromere (Fig. 3).

\section{HASPIN-DEPENDENT H3 PHOSPHORYLATION REQUIRED FOR CPC TARGETING TO CENTROMERES}

In human cells, Haspin, a histone kinase that phosphorylates threonine 3 of $\mathrm{H} 3$, has been suggested to function in the centromeric protection of cohesin in parallel with shugoshin (Dai et al. 2006). It turns out that fission yeast Haspin-related kinase Hrk1 is dispensable for centromeric cohesion or Sgo1-related function but required for CPC targeting to centromeres, a function related to Sgo2 (Yamagishi et al. 2010). In fission yeast, Hrk1 phosphorylates $\mathrm{H} 3-\mathrm{T} 3$ only in M phase, and this phosphorylation is re-
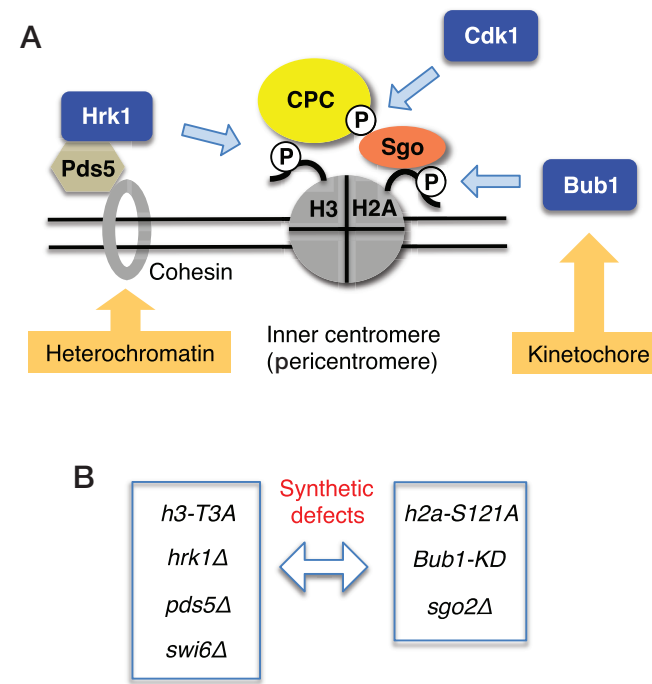

Figure 4. $(A) \mathrm{CPC}$ targeting to centromeres and $(B)$ genetic interaction of two histone phosphorylation pathways. $(A)$ Whereas $\mathrm{H} 3-\mathrm{pT} 3$ associates with $\mathrm{CPC}, \mathrm{H} 2 \mathrm{~A}-\mathrm{pS} 121$ facilitates interaction of the nucleosome with shugoshin. Cdk1-dependent phosphorylation of CPC promotes binding to shugoshin, and neither protein can fully localize to chromatin without this phosphorylation (Tsukahara et al. 2010), suggesting that physical interaction between CPC and shugoshin creates synergetic association of these proteins with nucleosomes. Thus, phosphorylation of CPC contributes to chronological regulation of centromere targeting of $\mathrm{CPC}$ during the cell cycle, whereas two histone marks contribute to special regulation of the CPC. $(B)$ Mutations in the two histone phosphorylation pathways cause no cumulative defects if combined within the intrapathway, whereas interpathway double mutations cause synthetic defects.

quired for full localization of the CPC to centromeres. H3T3 interacts directly with the conserved BIR domain of Survivin, and mutational analysis of the BIR domain indicates that this interaction is important for CPC targeting to centromeres (Fig. 4A). This mechanism is conserved in vertebrates (Kelly et al. 2010; Wang et al. 2010; Yamagishi et al. 2010). Genetic analyses in fission yeast reveal that this Hrk1-H3(pT3) pathway by which the CPC is localized to centromeres acts in a redundant capacity with the Bub1H2A(pS121)-Sgo2 pathway; intrapathway double mutations cause no cumulative defects, whereas interpathway double mutations cause remarkable defects in chromosome biorientation (Fig. 4B). Importantly, the biorientation defects of double mutants such as $h 3-T 3 A$ and $h 2 a-S 121 A$ are largely suppressed by ectopically localizing the $\mathrm{CPC}$ to centromeres. Thus, the primary readout of histone phosphorylation of H3-T3 and H2A-S121 is the localization of the $\mathrm{CPC}$ to the inner centromere, a process essential for setting up chromosome biorientation (Yamagishi et al. 2010).

Although Bub1 and Hrk1 kinases cooperate in targeting the $\mathrm{CPC}$ to centromeres, the activities of these kinases are distributed differently along the chromosome in fission yeast; Bub1 locates at kinetochores (Kawashima et al. 2010), whereas Hrk1 interacts with the cohesin-associating protein Pds5 and locates at cohesin sites along the 


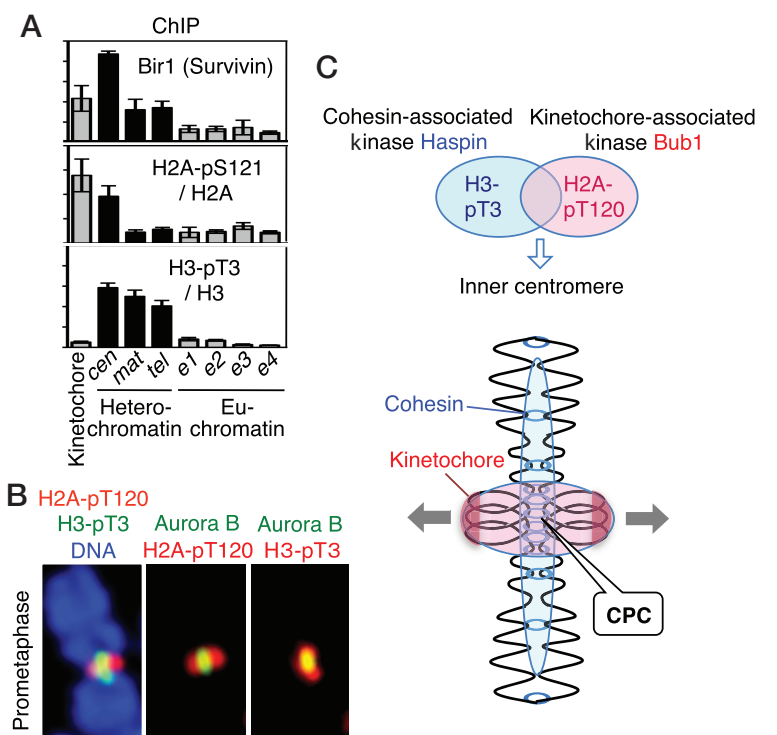

Figure 5. Intersection of two histone marks defines inner centromere. (A) Chromatin immunoprecipitation (ChIP) analysis of Survivin/Bir1, Hs-pT3, and H2A-pS121 throughout the kinetochore. (cen) Heterochromatic centromere, (mat) mating-type locus, (tel) telomere, and (el-4) euchromatic arm region in mitotic fission yeast cells. (B) Prometaphase chromosome from HeLa cells stained with antibodies against H3-pT3, H2A-pT120, and Aurora B. DNA was costained with Hoechst 33342. (C) Depiction of a chromosome and definition of inner centromere. (Modified, with permission, from Yamagishi et al. 2010 [C AAAS]).

chromosome. Accordingly, H2A-pT121 is enriched at the core centromere and the adjacent pericentromeric region (the inner centromere in metazoans), whereas H3-pT3 distributes to heterochromatic regions where cohesin is enriched. The CPC localizes largely at the pericentromeric region, the intersection of H3-pT3 and H2A-pS121 distributions (Fig. 5A) (Yamagishi et al. 2010). In human prometaphase, chromosome H3-pT3 signals localize to the intersister region, whereas H2A-pT120 (equivalent to yeast H2A-pS121) signals locate along the interkinetochore axis, with signals culminating near kinetochores. Importantly, Aurora B signals are enriched in the merged region of these signals (Fig. 5B). Thus, the inner centromere in eukaryotes is principally defined by the intersection of two histone marks, H2A-pS121 and H3-pT3, that are mediated by kinetochore-associated kinase Bub1 and cohesin-associated kinase Haspin/Hrk1 (Fig. 5C) (Yamagishi et al. 2010).

\section{CONCLUSIONS}

The CPC has several noncentromeric roles during prophase such as phosphorylation of H3-S10 and localization of condensin onto global chromatin. In animal cells, CPC localization is dynamically narrowed down to the inner centromere during prometaphase (Ruchaud et al. 2007). For this redistribution, Bub1 kinase locating at kinetochores has a central role by localizing H2A-pS121 and shugoshin near the centromere (Kiyomitsu et al. 2007;
Kawashima et al. 2010). In addition to CPC targeting, shugoshin has a crucial role in protecting cohesin from the prophase dissociation pathway (Salic et al. 2004; Kitajima et al. 2005; McGuinness et al. 2005), which may lead to the enrichment of cohesin and thereby the enrichment of Haspin and H3-pT3 at centromeres. This will further promote the centromeric localization of the shugoshin-CPC, thus constructing a positive-feedback loop between cohesin and shugoshin at centromeres. In fact, localization interdependencies are observed not only between shugoshin and the CPC but also between shugoshin and cohesin in several organisms (Hamant et al. 2005; Kiburz et al. 2005; Dai et al. 2006; Resnick et al. 2006; Kawashima et al. 2007; Rivera and Losada 2009; Shintomi and Hirano 2009). Thus, the newly uncovered molecular network centered on cohesin-shugoshin-CPC has a fundamental role in coupling sister chromatid cohesion to chromosome biorientation in eukaryotes (Yamagishi et al. 2010).

\section{ACKNOWLEDGMENTS}

Y.W. thanks past and present members of the laboratory for contributing to the research cited in this chapter. Research is supported by a Grant-in-Aid for Specially Promoted Research from the Ministry of Education, Culture, Sports, Science and Technology (MEXT), Japan.

\section{REFERENCES}

Boyarchuk Y, Salic A, Dasso M, Arnaoutov A. 2007. Bub1 is essential for assembly of the functional inner centromere. J Cell Biol 176: 919-928.

Cheeseman IM, Chappie JS, Wilson-Kubalek EM, Desai A. 2006. The conserved KMN network constitutes the core microtubulebinding site of the kinetochore. Cell 127: 983-997.

Chen Q, Zhang X, Jiang Q, Clarke PR, Zhang C. 2008. Cyclin B1 is localized to unattached kinetochores and contributes to efficient microtubule attachment and proper chromosome alignment during mitosis. Cell Res 18: 268-280.

Ciferri C, Pasqualato S, Screpanti E, Varetti G, Santaguida S, Dos Reis G, Maiolica A, Polka J, De Luca JG, De Wulf P, et al. 2008. Implications for kinetochore-microtubule attachment from the structure of an engineered Ndc80 complex. Cell 133: 427-439.

Dai J, Sullivan BA, Higgins JMG. 2006. Regulation of mitotic chromosome cohesion by haspin and Aurora B. Dev Cell 11: $741-750$

DeLuca JG, Gall WE, Ciferri C, Cimini D, Musacchio A, Salmon ED. 2006. Kinetochore microtubule dynamics and attachment stability are regulated by Hec1. Cell 127: 969-982.

Hamant O, Golubovskaya I, Meeley R, Fiume E, Timofejeva L, Schleiffer A, Nasmyth K, Cande WZ. 2005. A REC8-dependent plant shugoshin is required for maintenance of centromeric cohesion during meiosis and has no mitotic functions. Curr Biol 15: $948-954$.

Hauf S, Cole RW, LaTerra S, Zimmer C, Schnapp G, Walter R, Heckel A, van Meel J, Rieder CL, Peters J-M. 2003. The small molecule Hesperadin reveals a role for Aurora B in correcting kinetochore-microtubule attachment and in maintaining the spindle assembly checkpoint. J Cell Biol 161: 281-294.

Hauf S, Roitinger E, Koch B, Dittrich CM, Mechtler K, Peters JM. 2005. Dissociation of cohesin from chromosome arms and loss of arm cohesion during early mitosis depends on phosphorylation of SA2. PLoS Biol 3: e69.

Indjeian VB, Stern BM, Murray AW. 2005. The centromeric protein Sgol is required to sense lack of tension on mitotic chromosomes. Science 307: 130-133. 
Jeyaprakash AA, Klein UR, Lindner D, Ebert J, Nigg EA, Conti E. 2007. Structure of a Survivin-Borealin-INCENP core complex reveals how chromosomal passengers travel together. Cell 131: 271-285.

Kawashima SA, Tsukahara T, Langegger M, Hauf S, Kitajima TS, Watanabe Y. 2007. Shugoshin enables tension-generating attachment of kinetochores by loading Aurora to centromeres. Genes Dev 21: 420-435.

Kawashima SA, Yamagishi Y, Honda T, Ishiguro K, Watanabe Y. 2010. Phosphorylation of H2A by Bub1 prevents chromosomal instability through localizing shugoshin. Science 327: 172-177.

Kelly AE, Funabiki H. 2009. Correcting aberrant kinetochore microtubule attachments: An Aurora B-centric view. Curr Opin Cell Biol 21: 51-58.

Kelly AE, Ghenoiu C, Xue JZ, Zierhut C, Kimura H, Funabiki H. 2010. Survivin reads phosphorylated histone $\mathrm{H} 3$ threonine 3 to activate the mitotic kinase Aurora B. Science 330: 235-239.

Kiburz BM, Reynolds DB, Megee PC, Marston AL, Lee BH, Lee TI, Levine SS, Young RA, Amon A. 2005. The core centromere and Sgol establish a 50-kb cohesin-protected domain around centromeres during meiosis I. Genes Dev 19: 3017-3030.

Kitajima TS, Kawashima SA, Watanabe Y. 2004. The conserved kinetochore protein shugoshin protects centromeric cohesion during meiosis. Nature 427: 510-517.

Kitajima TS, Hauf S, Ohsugi M, Yamamoto T, Watanabe Y. 2005. Human Bub1 defines the persistent cohesion site along the mitotic chromosome by affecting shugoshin localization. Curr Biol 15: $353-359$.

Kitajima TS, Sakuno T, Ishiguro K, Iemura S, Natsume T, Kawashima SA, Watanabe Y. 2006. Shugoshin collaborates with protein phosphatase 2A to protect cohesin. Nature 441: 46-52.

Kiyomitsu T, Obuse C, Yanagida M. 2007. Human Blinkin/AF15q14 is required for chromosome alignment and the mitotic checkpoint through direct interaction with Bub1 and BubR1. Dev Cell 13: 663-676.

Lee JY, Hayashi-Hagihara A, Orr-Weaver TL. 2005. Roles and regulation of the Drosophila centromere cohesion protein MEIS332 family. Philos Trans R Soc Lond B Biol Sci 360: 543-552.

Liu D, Vader G, Vromans MJ, Lampson MA, Lens SM. 2009. Sensing chromosome bi-orientation by spatial separation of aurora B kinase from kinetochore substrates. Science 323: 1350 1353.

Losada A, Hirano M, Hirano T. 2002. Cohesin release is required for sister chromatid resolution, but not for condensin-mediated compaction, at the onset of mitosis. Genes Dev 16: 3004-3016.

Marston AL, Tham WH, Shah H, Amon A. 2004. A genome-wide screen identifies genes required for centromeric cohesion. Science 303: 1367-1370.

McGuinness BE, Hirota T, Kudo NR, Peters J-M, Nasmyth K 2005. Shugoshin prevents dissociation of cohesin from centromeres during mitosis in vertebrate cells. PLoS Biol 3: e86.

Miyazaki WY, Orr-Weaver TL. 1994. Sister-chromatid cohesion in mitosis and meiosis. Annu Rev Genet 28: 167-168.

Nasmyth K, Peters J-M, Uhlmann F. 2000. Splitting the chromosome: Cutting the ties that bind sister chromatids. Science $\mathbf{2 8 8}$
1379-1385

Pinsky BA, Biggins S. 2005. The spindle checkpoint: Tension versus attachment. Trends Cell Biol 15: 486-493.

Rabitsch KP, Gregan J, Schleiffer A, Javerzat JP, Eisenhaber F, Nasmyth K. 2004. Two fission yeast homologs of Drosophila MeiS332 are required for chromosome segregation during meiosis I and II. Curr Biol 14: 287-301.

Resnick TD, Satinover DL, MacIsaac F, Stukenberg PT, Earnshaw WC, Orr-Weaver TL, Carmena M. 2006. INCENP and Aurora B promote meiotic sister chromatid cohesion through localization of the Shugoshin MEI-S332 in Drosophila. Dev Cell 11: $57-68$.

Riedel CG, Katis VL, Katou Y, Mori S, Itoh T, Helmhart W, Galova M, Petronczki M, Gregan J, Cetin B, et al. 2006. Protein phosphatase $2 \mathrm{~A}$ protects centromeric sister chromatid cohesion during meiosis I. Nature 441: 53-61.

Rivera T, Losada A. 2009. Shugoshin regulates cohesion by driving relocalization of PP2A in Xenopus extracts. Chromosoma 118: 223-233.

Ruchaud S, Carmena M, Earnshaw WC. 2007. Chromosomal passengers: Conducting cell division. Nat Rev Mol Cell Biol 8: 798-812.

Salic A, Waters JC, Mitchison TJ. 2004. Vertebrate shugoshin links sister centromere cohesion and kinetochore microtubule stability in mitosis. Cell 118: $567-578$.

Shintomi K, Hirano T. 2009. Releasing cohesin from chromosome arms in early mitosis: Opposing actions of Wapl-Pds5 and Sgo1. Genes Dev 23: 2224-2236.

Tanaka TU. 2008. Bi-orienting chromosomes: Acrobatics on the mitotic spindle. Chromosoma 117: 521-533.

Tang Z, Shu H, Qi W, Mahmood NA, Mumby MC, Yu H. 2006. PP2A is required for centromeric localization of Sgol and proper chromosome segregation. Dev Cell 10: 575-585.

Tanno Y, Kitajima TS, Honda T, Ando Y, Ishiguro K, Watanabe Y. 2010. Phosphorylation of mammalian Sgo2 by Aurora B recruits PP2A and MCAK to centromeres. Genes Dev 24: 2169 2179.

Tsukahara T, Tanno Y, Watanabe Y. 2010. Phosphorylation of the $\mathrm{CPC}$ by $\mathrm{Cdk} 1$ promotes chromosome bi-orientation. Nature 467: 719-723.

Vanoosthuyse V, Prykhozhij S, Hardwick KG. 2007. Shugoshin 2 regulates localization of the chromosomal passenger proteins in fission yeast mitosis. Mol Biol Cell 18: 1657-1669.

Wang F, Dai J, Daum JR, Niedzialkowska E, Banerjee B, Stukenberg PT, Gorbsky GJ, Higgins JM. 2010. Histone H3 Thr-3 phosphorylation by Haspin positions Aurora B at centromeres in mitosis. Science 330: 231-235.

Watanabe Y. 2005. Shugoshin: Guardian spirit at the centromere. Curr Opin Cell Biol 17: 590-595.

Yamagishi Y, Sakuno T, Shimura M, Watanabe Y. 2008. Heterochromatin links to centromeric protection by recruiting shugoshin. Nature 455: 251-255.

Yamagishi Y, Honda T, Tanno Y, Watanabe Y. 2010. Two histone marks establish the inner centromere and chromosome bi-orientation. Science 330: 239-243. 


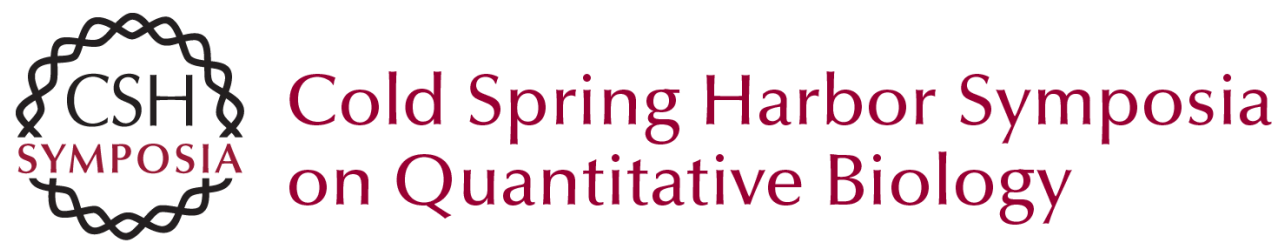

\section{Temporal and Spatial Regulation of Targeting Aurora B to the Inner Centromere}

Y. Watanabe

Cold Spring Harb Symp Quant Biol 2010 75: 419-423 originally published online March 29, 2011 Access the most recent version at doi:10.1101/sqb.2010.75.035

References This article cites 44 articles, 16 of which can be accessed free at: http://symposium.cshlp.org/content/75/419.full.html\#ref-list-1

\section{License}

Email Alerting Service top right corner of the article or click here.

To subscribe to Cold Spring Harbor Symposia on Quantitative Biology go to: http://symposium.cshlp.org/subscriptions 